Naturwissenschaften 82, 432-434 (1995) (C) Springer-Verlag 1995

\section{Vision Through Colored Eyes}

\author{
K. Lunau, H. Knüttel \\ Institut für Zoologie der Universität, D-93040 Regensburg, Germany
}

The conspicuous eye-color patterns of tabanid flies have been frequently portrayed. The eye colors originate from cornea color filters, which, on the one hand, cause colorful reflections and, on the other, alter the spectral composition of transmitted light. In this study, spectral transmission measurements of single cornea lenses were performed in order to study the visual function of cornea color filters. Spectral transmission curves allow a quantitative description of the difference between input stimulus and utilized stimulus caused by the transmission properties of this stimulus-conducting system.

The external appearance of dipteran compound eyes can be explained by different reasons: 1) The eye pigments, viewed through the dioptric apparatus, result in dull colors which are usually reddish or brownish in flies [1]. 2) Layered cornea lenses cause colorful metallic reflections or interference colors $[2-4,22]$ so that additional reflections from eye pigments may not be recognized for the most part. 3) The cuticle of facet intersections causes reflections of pigment colors of interference colors. In most Diptera, the color of the compound eyes is rather uniform and dull. However, metallic green and even metallic multicolored compound eyes have been reported for 72 species out of 23 families of Diptera [5, 6]. Phasecontrast light microscopy and transmission electron microscopy of the metallic eyes of tabanid flies demonstrated the presence of a multiple layering of alternating layers of high and low refractive index near the front surface of the cornea lens. The variation in color and intensity of the reflections depends on the angles of viewing and illumination. The measured reflection properties of single cornea lenses of intact eyes corresponded to calculated reflection properties, which were based on the assumption that the layer systems function as interference filters composed of quarterwavelength layers $[2,3,7]$. This study examines the spatial arrangement of the various colored facets in four species with very conspicuous eye-color patterns: three tabanid flies (Chrysops relictus Meigen, Haematopota pluvialis L., Heptatoma pellucens F.; Tabanidae, Diptera) and one dolichopodid fly (Hercostomus germanus Wiedemann; Dolichopodidae, Diptera), and presents spectral transmission curves of single cornea lenses.

The external appearance of the colored eye patterns was described by means of observations of living specimens with a Wild M3B stereomicroscope under various illuminations. The spectral transmission of single cornea lenses was recorded with a Bentham spectrometer system (monochromator M 300EA, photomultiplier DH3) which was combined with a UV-transmitting objective (Zeiss Ultrafluar $32 \times$ ). The measuring aperture $(\phi 0.12 \mathrm{~mm})$ was adjusted to the facet center. Illumination was provided by a $75-W$ Zeiss Xenon lamp. The light beam was adjusted at right angles to the facet surface and to the objective surface. The diameter of the measuring spot amounted to $10 \mu \mathrm{m}$. Raw data were taken at 5 -nm increments between limits of 265 and $800 \mathrm{~nm}$. For the preparation of isolated corneas, living flies were decapitated. The cornea was prepared in fly Ringer solution (150 $\mathrm{m} M \mathrm{NaCl}, 10 \mathrm{~m} M \mathrm{KCl}, 2 \mathrm{~m} M$ $\mathrm{CaCl}_{2}$. Fixation may strongly influence the transmission properties of cornea lenses $[8,9]$. A small retouching brush was used to clean the inner cornea surface. Several cuts were made into the cornea, before it was spread in fly Ringer solution between two cover slips which were mounted on the opening of a slide. Water evaporation was prevented by sealing with Vaseline. The measuring light transmitted the top cover slip, Ringer solution, the facet center in the physiological orthodrome direction, Ringer solution, and the bottom cover slip. Reference measurements were taken from the same preparation, each in close proximity to the original measurement, but without cornea facets in the light path. The spectral transmission was calculated from original and reference measurements. In order to reduce spectral artifacts, the light path was carefully adjusted at right angles to the very center of the facets. Only minor effects were found if this method was systematically varied in order to trace spectral artifacts.

The spatial arrangement of colored cornea lenses differs greatly between the tested species. The spectral transmission of cornea lenses corresponding to dark brown facets in the three tabanid species is approx. $100 \%$ throughout the range of wavelengths tested, and has no distinct minimum. The human-visible color of the metallic, colored facets corresponds to the wavelength position of the transmission minimum, as if looking at the reflection and transmission of interference filters. The spectral transmission curves of the main types of cornea lenses in the species tested are shown in Fig. 1. Measuring very fresh preparations, the calculated values of spectral transmission often surpass the $100 \%$ level which is intrinsic to the focusing effect of the lenses.

The bright green-colored compound eye of female Chrysops relictus has four dark brown round areas each covering approx. 50 facets and bordered by reddish facets. In males, the spatial pattern is female-like, but reduced in size, and shifted to the ventral half of the compound eye. The dorsal half of male compound eyes shows uniform bright green reflections. The spectral transmission curve of bright green facets has a minimum at $570 \mathrm{~nm}$ with a half-intensity bandwidth of approx. $60-70 \mathrm{~nm}$. Transmission at the minimum is reduced by up to $50 \%$, as compared to that at wavelengths shorter than $520 \mathrm{~nm}$ and longer than $620 \mathrm{~nm}$. The minima of spectral transmission curves of reddish facets are less pronounced and their positions have shifted to longer wavelengths. The spectral transmission of green facets of the dorsal eye region of males differs only slightly from that of green facets in the ventral eye, and from that of green facets of conspecific females (Fig. 1A). The green/orange eyes of female Haematopota pluvialis have four hori- 

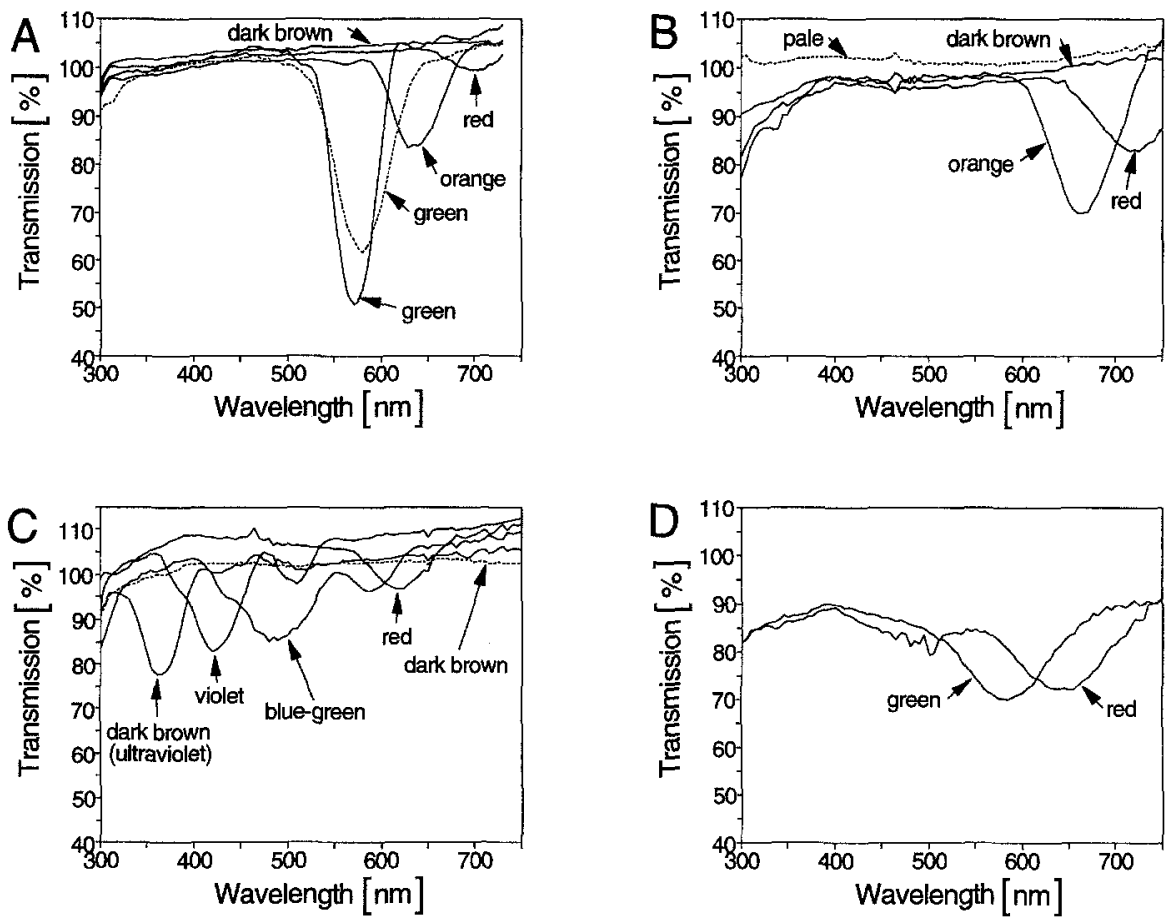

Fig. 1. Spectral transmission curves of single cornea lenses. Each figure shows a typical set of spectral transmission curves. Labels indicate the human-visible color of the facet. Reflection values $>100 \%$ are caused presumably by the light-collecting properties of the cornea lenses. A) Variously colored facets of a female Chrysops relictus (solid lines). Almost identical curves were found in male conspecifics. Facet of the green male-specific dorsal eye region (dotted line). B) Variously colored facets of a female Haematopota pluvialis (solid lines). Almost identical curves were found in male conspecifics. Facet of the pale male-specific dorsal eye region (dotted line). C) Variously colored facets of a female Heptatoma pellucens. D) Red and green facets of a female Hercostomus germanus. Almost identical curves were found in male conspecifics

zontal irregular dark brown stripes. The green/orange eye regions consist of a number of facets almost equal to the dark brown eye regions. Intermediate areas of red facets are small in extension. The spatial pattern of males is female-like, but reduced in size, and shifted to the ventral half of the compound eye. The dorsal half of males' compound eyes is uncolored and pale [25]. The spectral transmission curve of orange facets has a minimum at $650 \mathrm{~nm}$ with a half-intensity bandwidth of approx. $100 \mathrm{~nm}$. The minima of the spectral transmission curves of reddish facets are less pronounced and their positions have shifted to longer wavelengths. Males show a constantly high spectral transmission of the pale facets in the male-specific dorsal eye region, similar to that of the dark brown facets in both sexes (Fig. 1 B).

Female Heptatoma pellucens have four bright, colorful horizontal areas in their dark brown eyes, each showing a rainbow-like color pattern. The dorsalmost stripe shows a simple rainbowlike color pattern, whereas the others show colorful reflections resembling a symmetrical double rainbow of red to green to blue to green to red. The wavelength positions of the transmission minima change step by step from ultraviolet to red, and additional minima occur. The transmission minima are more pronounced at shorter wavelengths; the decrease of the spectral transmission curve of minima in the ultraviolet is approx. $25 \%$, that of mini$m a$ in the red range of wavelengths approx. $10 \%$ (Fig. 1C).

The eye color pattern of Hercostomus germanus (Dolichopodidae) is not sexspecific. Males and females exhibit a very regular vertical row-by-row color pattern of red and green cornea facets in the frontal and ventral parts of the compound eyes, which is also typical for other species of the subfamily Dolichopodinae [29], e.g., Poecilobo- thrus nobilitatus L. and Dolichopus discifer Stannius [9]. The entire compound eye has colored cornea lenses. The two types of spectral transmission curves show one moderate minimum each. Green facets show a minimum at $570 \mathrm{~nm}$, red facets at $650 \mathrm{~nm}$ (Fig. 1D). The measurements of the spectral transmission of single cornea lenses demonstrate that metallic, colored cornea facets modify the spectral composition of transmitted light, whereas dark brown cornea lenses do not. As an exception, some dorsalmost dark brown cornea lenses in $H$. pellucens show a reduced transmission in the ultraviolet range of wavelengths (Fig. 1C). In this case, the corresponding $\mathrm{UV}$ reflection is invisible to humans.

The significance of the colored cornea lenses in the compound eyes of flies is still hypothetical. The corneal interference filters alter the reflection properties as well as the transmission properties of the eyes. Therefore, one has to consider their function for a signal sender as well as their function for a receiver of visual information. On the one hand, it is possible that the external appearance of compound eyes, caused by the colorful reflections from the facets, serves as an intraspecific communication signal. On the other hand, it is tempting to ask whether the flies could benefit from the wavelength-specific, reduced transmission in terms of an improved perception of visual signals [2]. In order to discuss this possibility, we would like to highlight some important details about the intraspecific communication by visual signals in these flies. Both dolichopodid and tabanid flies have black-and-white ornamentations of wings, legs, abdomen, head, or antennae. For Dolichopodidae only, it was shown that males display these signals during courtship [16, 17]. It is not known whether tabanid flies also communicate by means of their conspicuous black-and-white signals. Courtship of most dolichopodid flies occurs among the foliage of reeds, herbs, or trees, or under the tree canopy. During premating behavior, male tabanid flies prefer to hover in open spaces around the tree canopy in the early morning (sunrise) $[14,15,23,24]$ or near ground level in shafts of sunlight $[20,21,23]$, then pursue and copulate with passing females. The spectral composition of 
ambient light is strongly dependent on the habitat and time of day [13]: in a canopy habitat or among green leaves, ambient light is dominated by wavelengths longer than $520 \mathrm{~nm}$, due to the reflection and transmission properties of green foliage; during sunrise and sunset, it is dominated by blue and red wavelengths, since the open sky is dominated by blue wavelengths.

A possible function of the colored cornea lenses might be the screening against background light, which is useful if bright objects are viewed against a colored background. The perceptual contrast between object and background is improved if the wavelength position of the background color's reflection peak corresponds to that of the transmission minimum of the cornea lens.

The selective spectral transmission could also affect the steady state of rhodopsin molecules: because rhodopsins and corresponding metarhodopsins show different spectral sensitivities, the photoreconversion of metarhodopsins is dependent on the spectral composition of incident light [11, 18]. Rhodopsin molecules of the short-, middle-, and long-wavelength types of photoreceptors in the insects tested maximally absorb at approx. 350,440 , and $530 \mathrm{~nm}$, respectively, whereas most of the corresponding metarhodopsins maximally absorb in the small range of wavelengths between 470 and $500 \mathrm{~nm}$ [11]. Our preliminary studies suggest that, in the tabanid and dolichopodid flies tested, the wavelength-selective transmission seems to be well tuned to the wavelength-selective photoreconversion in such a manner that it could yield a photosteady state with a high rhodopsin content. Hitherto, we have not yet found a corneal lens with a transmission minimum corresponding to the range of wavelengths in which many metarhodopsins maximally absorb.

The spectral sensitivity of tabanid and dolichopodid photoreceptors has not been studied extensively. However, morphological studies show that Tabanidae and Dolichopodidae and other families have inherited the same trapezoidal pattern of rhabdomeres in the ommatidia of the compound eye known from modern muscoid families $[3,22$, 26-28]. The R 8y type of photoreceptor, described as a component of the apposition subsystem in muscoid flies, is sensitive in the green/yellow waveband [12]. If such a receptor is present in Dolichopodidae and Tabanidae, it is a candidate type of photoreceptor which might be affected by the change of the spectral composition of light via green or orange cornea lenses. The reduced transmission of ultraviolet or blue light by cornea filters may serve analogous functions in other types of photoreceptors.

In the case of Poecilobothrus nobilitatus, it was suggested that the two cornea filters differing in the wavelength position of their transmission minimum might be adapted to the perception of the body color originating from the metallic colored cuticle [19]. Because the body color regularly varies from green in the center to red in the periphery under field conditions, the central area of the body would appear darker if viewed through green facets, and brighter if viewed through red facets, as compared to the peripheral body area. This interpretation, however, cannot be applied to Tabanidae, because they do not have metallic interference body colors, unlike many Dolichopodidae. The male-specific dorsal eye region in tabanids presumably serves the detection of females from a great distance. The differences in the spectral transmission of the facets in the male-specific eye region in $H$. pluvialis and $C$. relictus suggest that the corneal filters could be adapted to habitat conditions.

We would like to hypothesize that colored cornea interference filters might improve the perception of blackand-white intraspecific communication signals in the natural courtship habitat. In addition, under conditions where the intensity peak of ambient light corresponds to the absorbance peak of rhodopsin molecules, this mechanism could yield a high steady state of rhodopsin. The courtship scenarios in the tested flies support the idea that the colored eyes might play a role in the perception of black-and-white signals under extreme light conditions.
We thank Prof. Dr. R. Schwind for critical discussions, Dr. P. Schlecht for methodical advice, and H. Stellner for linguistic improvement.

Received June 6, 1995

1. Langer, H., in: Photoreceptor Optics, p. 429 (A.W. Snyder, R. Menzel, eds.). Berlin: Springer 1975

2. Bernard, G. D.: J. Insect Physiol. 17, 2287 (1971)

3. Trujillo-Cenoz, O., Bernard, G.D.: J. Ultrastruct. Res. 38, 149 (1972)

4. Miller, W.H., in: Handbook of Sensory Physiology VII/6A, p. 69 (H. Autrum, ed.). Berlin: Springer 1979

5. Steyskal, G.C.: Bull. Brooklyn entomol. Soc. 44, 163 (1949)

6. Steyskal, G.C.: ibid. 52, 89 (1957)

7. Bernard, G.D., Gemne G., Seitz, G., in: Handbook of Sensory Physiology VII/2, p. 357 (M.G.F. Fuortes, ed.). Berlin: Springer 1972

8. Kolb, G., Autrum, H., Eguchi, E.: Z. vergl. Physiol. 63, 434 (1969)

9. Lunau, K.: unpublished

10. Bernard, G.D., Miller, W. H.: Invest. Ophthal. 7, 416 (1968)

11. Stavenga, D. G.: Trends Neurosci. 15,213 (1992)

12. Hardie, R.C., in: Progress in Sensory Physiology, Vol. 5, p. 1. (D. Ottoson, ed.). Berlin: Springer 1985

13. Endler, J.A.: Ecol. Monogr. 63, 1 (1993)

14. Bailey, N.S.: Ann. Entomol. Soc. Am. 41, 403 (1948)

15. Matsumura, T.: Kontyû 52, 321 (1984)

16. Lunau, K.: Zool. Beitr. 34, 465 (1992)

17. Lunau, K., Knüttel H.: Verh. Dtsch. Zool. Ges. 87, 48 (1994)

18. Hamdorf, K., Rosner G.: J. Comp. Physiol. 86, 281 (1973)

19. Land, M. F.: ibid. A 173, 595 (1993)

20. Smith, S. M., Turnbull, D. A., Taylor P.D.: J. Insect Behav. 7, 355 (1994)

21. Wilkerson, R.C., Butler, J.F.: Ann. Entomol. Soc. Am. 77, 293 (1984)

22. Smith, W. C., Butler, J. F.: J. Insect Physiol. 37, 287 (1991)

23. Wilkerson, R.C., Butler, J.F., Pechuman, L. L.: Myia 3, 515 (1985)

24. Gaugler, R., Schutz, S.: J. Insect Behav. 2, 775 (1989)

25. Dietrich, W.: Z. wiss. Zool. 92, 465 (1909)

26. Wunderer, H., Seifert, P., Pilstl, F., Lange, A., Smola, U.: Naturwissenschaften 77, 343 (1990)

27. Shaw, R.S.: Brain Behav. Evol. 35, 107 (1990)

28. Burakova, O.V., Mazokhin-Porshnyakov, G. A.: Entomol. Rev. 61, 26 (1982)

29. Classification according to: Ulrich, H.: Bonn. zool. Beitr. 31, 385 (1980) 